\title{
CAPITULO 16
}

\section{LEVANTAMENTO DE ESCALAS PARA AVALIAÇÃO PSICOLÓGICA DA}

\author{
PERSONALIDADE
}

\author{
$\underline{\text { Nathália Bonugli Caurin }}{ }^{1}$, André Sousa Rocha ${ }^{2}$, João Victor Braz ${ }^{3}$, Carlos Eduardo da \\ Silva Barbosa ${ }^{4}$, Estephany Silva Braz ${ }^{5}$ \\ ${ }^{1}$ Universidade São Francisco, (nathaliacaurin@gmail.com) \\ ${ }^{2}$ Universidade São Francisco, (andresousarocha9@gmail.com) \\ ${ }^{3}$ Universidade São Francisco, (joaovictorbrazcontato@gmail.com) \\ ${ }^{4}$ Universidade do Grande Rio (UNIGRANRIO), (cedsbzs@gmail.com) \\ ${ }^{5}$ Universidade São Francisco, (estephanysilva14@hotmail.com)
}

\begin{abstract}
Resumo:
Objetivo: Realizar um levantamento de artigos que utilizaram escalas e instrumentos de avaliação da personalidade no contexto brasileiro nos últimos tempos. Método: Utilizou-se uma revisão narrativa da literatura de natureza qualitativa, descritiva e de levantamento. $\mathrm{O}$ estudo surgiu a partir do questionamento de quais escalas, instrumentos ou inventários estariam disponíveis para a avaliação psicológica da personalidade utilizadas em contexto brasileiro. Posteriormente, selecionaram-se as palavras-chave: cinco grandes fatores, traços de personalidade e inventário de personalidade acompanhadas do operador booleano and. As palavras-chave selecionadas foram aplicadas nas seguintes bases de dados e bibliotecas: Scientific Electronic Library On-line (SciELO), Biblioteca Virtual em Saúde (BVS) e Periódicos Eletrônicos de Psicologia (PEPSIC). Definiram-se como critérios de inclusão: artigos publicados no idioma português acessíveis na íntegra, sem especificação do recorte temporal. Excluíram-se estudos de revisão, literatura duplicada e repetida; anais publicados em eventos científicos, relatos de caso e de experiência, cartas ao editor, bem como outras literaturas que não passaram pelo processo rigoroso e sistemático de avaliação. Foram encontrados 667 estudos, sendo 399 na Scientific Electronic Library On-line (SciELO), 41 estudos na Literatura Latino-Americana e do Caribe em Ciências da Saúde (LILACS) e 227 estudos na Biblioteca Virtual em Saúde (BVS). Conforme os critérios de inclusão e exclusão, foram considerados os instrumentos contidos em 233 estudos para compor os resultados. Resultados e Considerações Finais: Os resultados apontaram para 28 instrumentos, comercializados ou não, utilizados para avaliação da personalidade, compreensão de psicopatologias, síndromes e sintomatologias relacionadas. Compreendeu-se a existência de escalas sendo desenvolvidas desde os anos 50, o que abrange a ideia de que a avaliação psicológica é uma das áreas mais antigas da psicologia e segue em constante crescimento, em específico a área da personalidade e suas novas propostas para abarcar as necessidades de instrumentos que avaliam tal medida.
\end{abstract}

Palavras-chave: Personalidade; Avaliação; Instrumentos; Escalas breves. Inovações 
Área Temática: Temas transversais - Outros.

E-mail do autor principal: nathaliacaurin@gmail.com

\section{INTRODUÇÃO}

Como área de conhecimento científico, a avaliação psicológica é uma das especialidades mais antigas da Psicologia. Isso porque a construção e o uso de instrumentos psicofísicos foram um dos marcos que permitiu a fundação de uma ciência psicológica que reunisse dados empíricos sobre os processos mentais, que se diferenciam da Biologia e Filosofia. Sendo assim, a avaliação psicológica é um campo de conhecimento, com pressupostos teóricos e metodológicos próprios e também uma prática profissional exclusiva do psicólogo que perpassa diversas áreas do campo psicológico (BUENO; PEIXOTO, 2018). Atualmente, é considerada também uma especialidade da Psicologia, conferida pelo Conselho Federal de Psicologia (CFP), o que reflete na demonstração dos avanços e das conquistas promovidas por essa área (WECHSLER; HUTZ; PRIMI, 2019).

Para realizar uma avaliação psicológica de qualidade, o psicólogo precisa, primeiramente, acolher e compreender a demanda. Após passar por essa análise inicial, terá capacidade de examinar as condições técnicas, éticas e científicas para prosseguir com a avaliação ou não. Em caso positivo, é preciso definir a finalidade da avaliação e levantar as hipóteses, selecionar os melhores instrumentos, técnicas e procedimentos para avaliar o fenômeno psicológico. Em caso negativo, é recomendado que o profissional encaminhe a avaliação para um profissional competente, ou seja, que tenha embasamento técnico e científico (REPPOLD; ZANINI; NORONHA, 2019; SCHNEIDER et al., 2021)

A personalidade se refere às diferenças individuais em padrões característicos de pensar, sentir e agir. Os estudos da personalidade focam em duas grandes áreas: uma é a compreensão das diferenças individuais em características que são peculiares da personalidade, como a irritabilidade e a sociabilidade. A outra é entender como as diversas partes de uma pessoa se unem como um todo (PIRES; NUNES; NUNES, 2019). Além disso, a personalidade diz respeito a atributos apresentados por cada sujeito, fazendo com que este seja único e, por meio das características individuais, o diferencie de todos os outros, corroborando para as diferentes manifestações de comportamentos, emoções e sentimentos (TRENTINI et al., 2009).

Um dos modelos mais reconhecidos na ciência psicológica para organizar hierarquicamente os traços de personalidade refere-se aos Cinco Grandes Fatores (CGF) (JOHN; NAUMANN; SOTO, 2008). A literatura apresenta o acrônimo OCEAN para melhor

\section{E - book Pesquisa e Tecnologia: Protagonismo e Inovações}


representar esses fatores, sendo: Openness to experience (abertura à experiência), Conscientiousness (conscienciosidade), Extraversion (extroversão), Agreeableness (amabilidade) e Neuroticism (neuroticismo).

A abertura a experiência se relaciona a apreciação a estética, a curiosidade intelectual e a imaginação criativa (COSTA; MCCRAE, 1992). A extroversão é marcada por cordialidade, sociabilidade e assertividade. A amabilidade está relacionada a polidez, a empatia e a compaixão. A conscienciosidade reflete características de organização, persistência e produtividade. Por fim, o neuroticismo está relacionado à depressão, à ansiedade e à volatilidade emocional (COSTA; MCCRAE, 1992; MCCRAE; COSTA, 2008; SOTO; JOHN, 2017)

Nesse sentido, diante da difusão, consolidação e replicação de diferentes estudos combinados com variadas culturas, ao longo tempo, foram elaborados instrumentos com a finalidade de avaliar traços da personalidade baseado no modelo dos Cinco Grandes Fatores (PIRES; NUNES; NUNES, 2019). Em relação aos contextos de aplicação da avaliação da personalidade, o levantamento realizado por Pires, Nunes e Oliveira em setembro de 2019 apontou que para as pesquisas realizadas no Brasil, os instrumentos têm sido utilizados para verificar associações entre a personalidade e os fenômenos da Psicologia Positiva, Organizacional, Educacional, construção e validação de testes, Saúde, Habilidades Sociais, risco no trânsito, conduta antissocial, conduta desviante, agressividade, cognição, escolha profissional, escolha do parceiro e ciúmes, esporte e segurança pública (PIRES; NUNES; OLIVEIRA, 2018).

Com este apanhado, designa-se o objetivo desta pesquisa. Visou-se um levantamento de artigos que utilizaram escalas e instrumentos de avaliação da personalidade no contexto brasileiro nos últimos tempos, apresentando quais escalas foram utilizadas e suas especificações, situando-se assim a recorrência de uso dessas escalas, estudos de tradução e adaptação e o desenvolvimento do conceito no decorrer do tempo até o presente momento.

\section{MÉTODO}

\section{Estratégia de busca}

Trata-se de um estudo de revisão narrativa de literatura de natureza qualitativa, descritiva e de levantamento. Para esse tipo de proposta metodológica não se exige a utilização de critérios sistemáticos e rigorosos para a busca de investigações na literatura (CORDEIRO,

\section{E - book Pesquisa e Tecnologia: Protagonismo e Inovações}


2007). Além disso, o processo de estratégia de busca para revisões narrativas não é exaustivo e sofisticado, o que sugere que o rastreio de artigos não precisa esgotar as fontes de informações. Portanto, a sua principal função é discutir o desenvolvimento ou o estado da arte de um determinado assunto, sob o ponto de vista teórico ou contextual (ROTHER, 2007).

O estudo surgiu a partir do questionamento de quais escalas, instrumentos ou inventários estariam disponíveis para a avaliação psicológica da personalidade utilizadas em contexto brasileiro. Posteriormente, após a definição do tema, selecionaram-se as seguintes palavraschave: Cinco Grandes Fatores, traços de personalidade e inventário de personalidade. Tais palavras-chave foram acompanhadas do operador booleano and, e o seu uso se justifica à medida que que não foram encontrados descritores controlados no descritores em Ciências da Saúde (DeCS).

De forma sucessiva, com a estratégia de busca definida, a pergunta de pesquisa idealizada, as palavras-chave selecionadas foram aplicadas nas seguintes bases/bancos de dados e bibliotecas: Scientific Electronic Library On-line (SciELO), Biblioteca Virtual em Saúde (BVS) e Periódicos Eletrônicos de Psicologia (PEPSIC).

\section{Critérios de Elegibilidade}

Os critérios de inclusão contemplaram artigos publicados no idioma portuguêsbrasileiro acessíveis na íntegra, sem especificação do recorte temporal, pois a intenção foi examinar as escalas disponíveis. Excluíram-se estudos de revisão, independente de sua natureza, ou seja, integrativa, sistemática ou metanálise; dissertações de mestrado e teses de doutorado, literatura duplicada e repetida; anais publicados em eventos científicos, cartas ao editor, bem como outras literaturas que não passaram pelo processo rigoroso e sistemático de avaliação. Outros fatores que foram considerados para remoção dos estudos: artigos em inglês ou espanhol e outras variáveis e escalas não disponíveis para uso.

Foram encontrados 667 estudos, sendo 399 na Scientific Electronic Library On-line (SciELO), 41 estudos na Literatura Latino-Americana e do Caribe em Ciências da Saúde (LILACS) e 227 estudos na Biblioteca Virtual em Saúde (BVS). Conforme os critérios de inclusão e exclusão, foram eliminadas 299 produções científicas na SicELO, 12 na Pepsic e 123 na BVS, totalizando 434 exclusões. Sendo assim, o que motivou a exclusão dos artigos foram: publicação em outro idioma, a saber, inglês e espanhol.

\section{E - book Pesquisa e Tecnologia: Protagonismo e Inovações}


Portanto, foram considerados os instrumentos contidos em 233 estudos para compor a análise e discussão. Sinaliza-se, que os estudos que apresentaram conteúdos com as mesmas escalas foram condensados em uma tabela na seção de resultados.

Tabela 1. Resultados encontrados de acordo com as palavras chaves utilizadas e suas bases de dados.

\begin{tabular}{|c|c|c|c|}
\hline Palavras-chave & Base/bancos de dados & Achados & Excluídos \\
\hline Cinco Grandes Fatores & Scielo & 84 & 59 \\
\hline Traços de Personalidade & Scielo & 185 & 144 \\
\hline Inventário de Personalidade & Scielo & 130 & 96 \\
\hline Cinco Grandes Fatores & PePSIC & 4 & 0 \\
\hline Traços de Personalidade & PePSIC & 37 & 12 \\
\hline Inventários de Personalidade & PePSIC & 0 & - \\
\hline Cinco Grandes Fatores & BVS & 70 & 68 \\
\hline Traços de Personalidade & BVS & 135 & 124 \\
\hline Inventários de Personalidade & BVS & 22 & 21 \\
\hline
\end{tabular}

Fonte: Elaborada pelos autores (2021).

\section{RESULTADOS E DISCUSSÃO}

Apresentam-se na tabela 1 os resultados obtidos do levantamento de escalas e instrumentos de personalidade que foram utilizados nos artigos selecionados. Na coluna 1, estão localizados os nomes dos instrumentos e suas siglas, na coluna 2 o nome dos autores que desenvolveram/adaptaram para o contexto brasileiro, e na coluna 3 as especificações de cada escala (quantidade de itens).

Tabela 2. Levantamento de escalas para avaliação psicológica da personalidade.

\begin{tabular}{|c|c|c|}
\hline Escala & Autores (as) & Especificações de cada escala \\
\hline $\begin{array}{l}\text { Inventário Multifásico Minnesota } \\
\text { de Personalidade (MMPI) }\end{array}$ & Hathaway e McKinley, 1951 & $\begin{array}{l}\text { Inventário originalmente americano, atualmente está } \\
\text { desfavorável para uso no Brasil. Conta com } 566 \\
\text { afirmações em versão longa, com } 10 \text { subescalas. }\end{array}$ \\
\hline Mini-Mult & Kincannon, 1968 & $\begin{array}{l}\text { Versão breve proposta a partir do MMPI. Conta com } \\
71 \text { itens que representam as } 10 \text { subescalas. }\end{array}$ \\
\hline $\begin{array}{l}\text { Eysenck Personality Questionnaire } \\
\text { Junior - EPQ-J \& adult (EPQ-A) }\end{array}$ & Eysenck \& Eysenck (1975/1998) & Conta com 81 itens. \\
\hline $\begin{array}{l}\text { Inventário dos Cinco } \\
\text { Fatores da Prande } \\
\end{array}$ & John, Donahue e Kentle (1991) & Conta com 44 itens. \\
\hline $\begin{array}{l}\text { Questionário de Dezesseis Fatores } \\
\text { de Personalidade (16PF) }\end{array}$ & Cattell, Cattell \& Cattell (1993) & Contém 185 itens. \\
\hline $\begin{array}{l}\text { Inventário fatorial de } \\
\text { personalidade (IFP) }\end{array}$ & Pasquali, Azevedo e Guesti (1997) & $\begin{array}{l}\text { Construído baseado na teoria das necessidades básicas } \\
\text { de Murray, composto por } 135 \text { itens. }\end{array}$ \\
\hline $\begin{array}{l}\text { Inventário Millon de Estilos de } \\
\text { Personalidade }\end{array}$ & $\begin{array}{l}\text { Millon (1997), adaptada para o } \\
\text { contexto brasileiro por Alchieri (2004) }\end{array}$ & Conta com 180 itens. \\
\hline $\begin{array}{l}\text { Marcadores para a avaliação da } \\
\text { personalidade no Modelo dos Cinco } \\
\text { Grandes Fatores }\end{array}$ & $\begin{array}{l}\text { Hutz, Nunes, Silveira, Serra, Anton e } \\
\text { Wieczorek (1998) }\end{array}$ & $\begin{array}{l}\text { Conta com } 96 \text { termos (adjetivos) que representam } 180 \\
\text { descritores de traços de personalidade. }\end{array}$ \\
\hline $\begin{array}{l}\text { Inventário de Personalidade Big } \\
\text { Five (IPBF) }\end{array}$ & $\begin{array}{l}\text { John \& Srivastava (1999), adaptado } \\
\text { para o contexto brasileiro por Barbosa } \\
(2009)\end{array}$ & Composto por 30 itens. \\
\hline $\begin{array}{l}\text { Escala Fatorial de Neuroticismo } \\
\text { (EFN) }\end{array}$ & Hutz \& Nunes (2001) & $\begin{array}{l}\text { Conta com } 82 \text { itens, com foco na avaliação de } \\
\text { ajustamento e instabilidade emocional. }\end{array}$ \\
\hline $\begin{array}{l}\text { ICFP- R (Instrumento dos Cinco } \\
\text { Fatores de Personalidade - } \\
\text { Reduzido) }\end{array}$ & $\begin{array}{l}\text { Troccóli,Vasconcelos, } \quad \text { Meiçó, } \quad \& \\
\text { Araújo, (2004) }\end{array}$ & Conta com 30 itens. \\
\hline
\end{tabular}




\begin{tabular}{|c|c|c|}
\hline $\begin{array}{l}\text { Inventário de Personalidade de } \\
\text { Freiburg (FPI-R) }\end{array}$ & $\begin{array}{l}\text { Soares, Machado, Dias, Pinho, Klein } \\
\text { (2004) }\end{array}$ & Composto por 138 itens. \\
\hline $\begin{array}{l}\text { Escala de Traços de Personalidade } \\
\text { para Crianças }\end{array}$ & Sisto (2004) & $\begin{array}{l}\text { Conta com } 30 \text { itens, para ser usado em crianças de } 5 \text { a } \\
10 \text { anos de idade. }\end{array}$ \\
\hline $\begin{array}{l}\text { Inventário De Cinco Fatores NEO } \\
\text { Revisado (Versão Curta) -(NEO- } \\
\text { FFI-R) }\end{array}$ & Flores-Mendoza (2007) & Conta com 60 itens. \\
\hline Escala Fatorial de Socialização & Nunes \& Hutz (2007) & $\begin{array}{l}\text { Conta com } 70 \text { itens, com foco na mensuração da } \\
\text { socialização, no modelo dos Cinco Grandes Fatores de } \\
\text { Personalidade. }\end{array}$ \\
\hline $\begin{array}{l}\text { Escala Fatorial de Extroversão } \\
\text { (EFEx) }\end{array}$ & Nunes \& Hutz (2007) & $\begin{array}{l}\text { Conta com } 57 \text { itens, com foco na mensuração da } \\
\text { extroversão, no modelo dos Cinco Grandes Fatores de } \\
\text { Personalidade. }\end{array}$ \\
\hline $\begin{array}{l}\text { Inventário dos Cinco } \text { Grandes } \\
\text { Fatores de Personalidade }- \text { IGFP-5 }\end{array}$ & Andrade (2008) & Medida de auto-relato breve, composta por 44 itens. \\
\hline \begin{tabular}{llr} 
Inventário & \multicolumn{2}{c}{ Dimensional dos } \\
Transtornos & da & Personalidade \\
(IDTP) & & \\
\end{tabular} & Carvalho (2008) & $\begin{array}{l}\text { Construído baseado na teoria de Millon. Conta com } \\
100 \text { itens. }\end{array}$ \\
\hline $\begin{array}{l}\text { Escala Fatorial de Abertura à } \\
\text { Experiência (EFA) }\end{array}$ & Vasconcellos \& Hutz, 2008 & $\begin{array}{l}\text { Conta com } 42 \text { itens, com foco na compreensão da } \\
\text { abertura à experiência, no modelo dos Cinco Grandes } \\
\text { Fatores de Personalidade. }\end{array}$ \\
\hline $\begin{array}{l}\text { Ten-Item Personality Inventory } \\
\text { (TIPI)-Br }\end{array}$ & Carvalho e Primi (2008) & $\begin{array}{l}\text { Composta por } 10 \text { itens, que são duplas de adjetivos e } \\
\text { que representam os traços de personalidade. }\end{array}$ \\
\hline $\begin{array}{l}\text { NEO de Personalidade Revisado } \\
\text { (NEO-PI-R) }\end{array}$ & Costa \& McCrae (2009) & Composto por 240 itens. \\
\hline $\begin{array}{l}\text { Inventário de Características da } \\
\text { Personalidade - ICP }\end{array}$ & Pinheiro, Gomes e Braga(2009) & Conta com 27 itens. \\
\hline $\begin{array}{l}\text { Bateria Fatorial de Personalidade } \\
\text { (BFP) }\end{array}$ & Nunes, Hutz \& Nunes (2010) & Composto por 126 itens. \\
\hline $\begin{array}{l}\text { Inventário Dimensional Clínico da } \\
\text { Personalidade (IDCP) }\end{array}$ & Carvalho e Primi (2011) & Contém 215 itens. \\
\hline $\begin{array}{l}\text { Inventário de } \begin{array}{c}\text { Organização de } \\
\text { Personalidade - Brasil (IPO-Br) }\end{array} \\
\text { (IPr) }\end{array}$ & Oliveira \& Bandeira (2011) & Conta com 83 itens. \\
\hline $\begin{array}{l}\text { Marcadores Reduzidos para } \\
\text { Avaliação da Personalidade-MRAP }\end{array}$ & Hauck et al. (2012) & $\begin{array}{l}\text { Avaliação com o público adolescente, composto por } 25 \\
\text { adjetivos que descrevem características estáveis da } \\
\text { personalidade. }\end{array}$ \\
\hline $\begin{array}{l}\text { Escala Reduzida de Descritores de } \\
\text { Personalidade - RED5 }\end{array}$ & Natividade \& Hutz (2015) & Consiste em 20 adjetivos ou pequenas expressões. \\
\hline $\begin{array}{l}\text { Inventário Dimensional Clínico da } \\
\text { Personalidade - versão triagem } \\
\text { (IDCP-triagem) }\end{array}$ & Carvalho, Pianowski, \& Reis, 2017 & $\begin{array}{l}\text { Desenvolvido com base na versão completa do IDCP } \\
\text { (Carvalho \& Primi, 2015), consiste em } 15 \text { itens. }\end{array}$ \\
\hline
\end{tabular}

Fonte: Elaborada pelos autores (2021).

Ao todo, foram achados 28 instrumentos. Alguns caíram em desuso (por exemplo Inventário Multifásico Minnesota de Personalidade) (HATHAWAY; MCKINLEY, 1951). No entanto, não perdem sua importância, pois foram fundamentais para o desenvolvimento e construção de instrumentos mais aprimorados com o avançar do tempo.

As escalas usadas de forma mais recorrente nas pesquisas foram: Inventário Dimensional Clínico da Personalidade - versão triagem (CARVALHO; PIANOWSKI; REIS, 2017). O estudo para desenvolver este instrumento contou com 1.196 pessoas, divididas entre grupo clínico e grupo não clínico. Tendo como base o Inventário Dimensional Clínico da Personalidade (CARVALHO; PRIMI, 2011), foi realizada uma análise de regressão logística e cálculo $d$ de Cohen, indicando os itens que melhor apontaram pessoas com ou sem transtornos de personalidade. No total, resultaram-se 15 itens com especificidade satisfatória para o uso em triagem (CARVALHO; PIANOWSKI; REIS, 2017).

\section{E - book Pesquisa e Tecnologia: Protagonismo e Inovações}


Seguindo com os inventários mais recorrentes, apresenta-se o Inventário de Organização de Personalidade - Brasil (IPO-Br). O instrumento foi adaptado e traduzido para uso na população brasileira, contando com 83 itens de autorrelato, que tem por objetivo avaliar funções psicológicas para o diagnóstico estrutural, segundo o Modelo de Organização da Personalidade (OLIVEIRA; BANDEIRA, 2011). O instrumento NEO de Personalidade Revisado (NEO-PIR) também demonstrou-se presente na busca, sendo um instrumento composto por 240 itens organizados e cinco subescalas, com o objetivo de rastrear a personalidade de seus respondentes, de acordo com a Teoria dos Cinco Grandes Fatores da Personalidade (Abertura, Conscienciosidade, Extroversão, Amabilidade e Neuroticismo) (COSTA; MCCRAE, 1982, 2008) .

Seguindo com o levantamento, o Inventário de Cinco Fatores Revisado - NEO-FFI-R, é um instrumento comercializado, e conta com 60 itens de questões afirmativas, baseadas nos Cinco Grandes Fatores, para avaliar a personalidade de adultos (FLORES-MENDOZA, 2007). Os Marcadores Reduzidos para Avaliação da Personalidade (MRAP) também foram localizados em diversos estudos, e tem o objetivo de avaliar a personalidade segundo os Cinco Grandes Fatores (CGF), através de 64 marcadores (adjetivos). A solução fatorial final deste instrumento conta com 25 marcadores, sendo cinco para cada área dos CGF (HAUCK-FILHO, MACHADO, TEIXEIRA; BANDEIRA, 2012).

A partir dos resultados levantados, entende-se por personalidade traços e diferenças individuais que caracterizam e descrevem um indivíduo. Esses traços evidenciam-se pela forma de se pensar, de agir, de sentir e de se expressar, e variam de pessoa para pessoa (PIRES; NUNES; NUNES, 2019). Apesar da variância individual, existem traços recorrentes nos perfis de personalidade, e uma das unidades de mensuração desses traços vem a ser o modelo baseado no Cinco Grandes Fatores (CGC), considerado atualmente o padrão ouro de reconhecimento de traços de personalidade. A partir da concordância acerca da elegibilidade destes fatores, muito criou-se e muito cria-se em decorrência dessa compreensão sobre a personalidade, havendo ampla pesquisa e desenvolvimento de escalas que buscam correlações entre os fatores de personalidade e uma infinitude de transtornos psicológicos, síndromes, sintomatologias e afins.

A avaliação da personalidade, seja ela partindo dos pressupostos da CGF ou não, fazem parte de uma universalidade mais extensa e mais antiga, a da avaliação psicológica. A avaliação psicológica é um campo complexo, amplo e sistêmico, que tem como e por finalidade a busca acerca do funcionamento psicológico das pessoas, utilizando-se de instrumentos padronizados e normativos de avaliação e medição e por outros meios passíveis de se alcançar a observação

\section{E - book Pesquisa e Tecnologia: Protagonismo e Inovações}


de teorias psicológicas em eventos observáveis (PRIMI, 2008; PRIMI, 2010). Pela amplitude e pela complexidade do intuito da avaliação psicológica, muitos são os instrumentos utilizados para se alcançar esta finalidade.

A partir do levantamento de escalas para avaliação psicológica dentro do âmbito da personalidade, notou-se a existência de escalas sendo desenvolvidas já em 1951. Tal fato reforça a ideia da preocupação com aspectos da personalidade e à atenção para aspectos relativos à mesma desde a primordialidade da psicologia compreendida como ciência (ABIB, 2009), consolidando-se como uma das especialidades mais antigas da psicologia (BUENO; PEIXOTO, 2018).

Por ser uma das áreas mais antigas, por conseguinte, acaba sendo também uma das mais revistas áreas da psicologia, fato este, passível de ser observado por meio da tabela acima exposta, demonstrando progressivo desenvolvimento de escalas baseadas nos CGF, e pela historicidade das evoluções, das novas adaptações e das presentes revisões. Tais avanços e contribuições consolidam-se por intermédio dos métodos desenvolvidos, pelas aplicações práticas passíveis de serem colhidas, pelos resultados obtidos e pelo devido reconhecimento cedido pelo Conselho Federal de Psicologia (CFP), que ao fazê-lo, atribui a devida cientificidade, e o devido crédito concedido pelos avanços apresentados (WECHSLER; HUTZ; PRIMI, 2019).

\section{CONSIDERAÇÕES FINAIS}

O principal objetivo do estudo foi fazer um levantamento, sem especificidade temporal, acerca dos instrumentos de avaliação da personalidade no Brasil. Acredita-se que o objetivo foi integralmente alcançado. A área da avaliação psicológica passou por intenso descrédito, sobretudo, nas décadas de 1970 e 1990, em consequência do mau uso das técnicas e instrumentos, que eram utilizados sem o devido cuidado quanto adaptação a realidade brasileira. Estudos dessa natureza visam reforçar o caráter ascendente e estável que a avaliação psicológica atingiu desde os anos 2000 (WECHSLER; HUTZ; PRIMI, 2019)

Nesse sentido, o presente estudo identificou, em sua maioria, instrumentos que utilizam como referencial teórico o modelo dos Cinco Grandes Fatores, teoria amplamente aceita por pesquisadores da avaliação da personalidade (SOTO; JOHN, 2017). Mais estudos podem ser realizados, de modo a buscar istrumentos que avaliem outros construtos, a fim de verificar o quão a área avançou nos últimos tempos.

As limitações podem ser endereçadas as bases e bibliotecas consultadas. Apesar do Inovações 
substancial número de publicações encontradas, a intenção do estudo não era esgotar a literatura que contém os instrumentos disponíveis para avaliar a personalidade. Contudo, acredita-se que a partir dos dados levantados, há uma variedade de instrumentos a serem utilizados em contextos diversos.

\section{REFERÊNCIAS}

ABIB, J. Epistemologia pluralizada e história da psicologia. Scientia e Studia, v. 7, n. 2, pp. $195-208,2009$

ALCHIERI, J. C. Modelo dos estilos de personalidade de Millon: adaptação do Inventário Millon de Estilos de Personalidade. Tese de Doutorado. Universidade Federal do Rio Grande do Sul, 2004.

ANDRADE, J. M de. Evidências de validade do inventário dos cinco grandes fatores de personalidade para o Brasil. Tese de Doutorado. Universidade de Brasília, Brasília, 2008.

BARBOSA, A. A. G. Modelo hierárquico de fobias infanto juvenis: testagem e relação com os estilos parentais. Tese de Doutorado. Universidade Federal da Paraíba, 2009.

BUEnO, J. M. H.; PEIXOTO, E. M. Avaliação Psicológica no Brasil e no Mundo. Psicologia: Ciência e Profissão, [S.L.], v. 38, pp. 108-121, 2018.

CATELL, R. B., CATELL, A. K. S. \& H. E. P. Questionário 16PF. Rio de Janeiro: CEPA, 1993.

CARVAlHO, L. de F. Construção e Validação do Inventário Dimensional dos Transtornos da Personalidade. Dissertação de Mestrado, Programa de Pós-Graduação Stricto Sensu em Psicologia, Universidade São Francisco, 2008.

CARVALHO, L. F; PRIMI, R. Desenvolvimento e investigação da estrutura interna do Inventário Dimensional Clínico da Personalidade (IDCP). Psicologia: Reflexão e Crítica, v. 28, pp. 322-330, 2011.

CARVALHO, L. F.; PRIMI, R. Versăo brasileira do Ten-Item Personality Inventory TIPIBr. Instrumento năo publicado, 2008.

CARVALHO, L. F.; PIANOWSKI, G.; REUS, A. M. Development and diagnostic accuracy of the screening of the Dimensional Clinical Personality Inventory. Psicologia: Ciência e Profissão, v. 37, n. 4, pp. 1011-1024, 2017.

CORDEIRO, A. M. et al. Revisão sistemática: uma revisão narrativa. Revista do Colégio Brasileiro de Cirurgiões, v. 34, p. 428-431, 2007.

COSTA, P. T.; MCCRAE, R. R. NEO-PI-R: Professional Manual, Florida: Psychological Assessment Resources Inc; 1992. Inovações 
COSTA, P. T.; McCRAE, R. NEO-PI-R - Inventário de Personalidade NEO Revisado Manual. São Paulo: Vetor, 2009.

EYSENCK, H. J.; EYSENCK, S. B. G. Manual of the Eysenck Personality Questionnaire (junior \& adult). Hodder and Stoughton Educational, 1975.

FLORES-MENDOZA, C. E. Estudo brasileiro do NEO-FFI-R (versão curta). In COSTA JUNIOR, P. T. \& McCRAE R. R. Avaliação em psicologia positiva NEO PI-R: Inventário de personalidade NEO revisado; e inventário de cinco fatores neo revisado: NEO-FFI-R (versão curta). São Paulo, SP: Vetor, pp. 93-98, 2007.

HAUCK-FILHO, N. et al. Evidências de validade de marcadores reduzidos para a avaliação da personalidade no modelo dos cinco grandes fatores. Psicologia: Teoria e Pesquisa, v. 28, n 4, pp. 417-423, 2012.

HATHAWAY, S. R., MCKINLEY, J. C. Inventário Multifásico Minnesota de Personalidade (MMPI). (Manual Técnico). Tradução e adaptação de BENKÖ, Antonius; SIMÕES, Roberto J.P., Rio de Janeiro: CEPA, 1970.

HUTZ, C. S.; NUNES, C. H. S. S. Escala fatorial de ajustamento emocional/neuroticismo (EFN). São Paulo: Casa do Psicólogo, 2001.

HUTZ, C. S. et al. O desenvolvimento de marcadores para a avaliação da personalidade no modelo dos cinco grandes fatores. Psicologia: reflexão e crítica, v. 11, pp. 395-411, 1998.

JOHN, O. P.; DONAHUE, E. M.; KENTLE, R. L. Big Five Inventory (BFI). University of California, Berkley, Institute of personality and social research, 1991.

JOHN, O. P.; SRIVASTAVA, S. The Big Five Trait taxonomy: History, measurement, and theoretical perspectives. In Handbook of personality: Theory and research. New York, NY: Guilford Press, pp. 102-138, 1999.

KINCANNON, J. C. Predição das pontuações da escala MMPI padrão de 71 itens: O Mini-Mult. Journal of Consulting and Clinical Psychology, v. 32, n. 3, pp. 319-325, 1968.

MCCRAE, R. R.; COSTA, P. T. The five-factor theory of personality. In O. P. John, R. W. Robins, \& L. A. Pervin (Eds.), Handbook of personality: Theory and research. The Guilford Press, pp. 159-181, 2008.

MILLON, M., WEISS, T.; LAWRENCE. MIPS Inventario Millon de estilos de personalidad. Buenos Aires: Paidós, 1997.

NATIVIDADE, J. C.; HUTZ, C. S. Escala Reduzida de Descritores dos Cinco Grandes Fatores de Personalidade: Prós e Contras. Psico, v. 46, n. 1, pp. 79-89, 2015.

NUNES, C. H. S. da S.; HUTZ, C. S. Construção e validação da escala fatorial de Socialização no modelo dos Cinco Grandes Fatores de Personalidade. Psicologia: Reflexão e Crítica, 20(1), pp. 20-25, 2007. Inovações 
NUNES, C. H. S. S. Lançamento da Escala Fatorial de Extroversão (EFEx) e Escala Fatorial de Socialização (EFS). Avaliação psicológica, v. 6, n. 1, pp. 103-106, 2007.

NUNES, C. H. S. S.; HUTZ, Claudio Simon; NUNES, Mariana Farias Oliveira. Bateria Fatorial de Personalidade (BFP): manual técnico. São Paulo: Casa do Psicólogo, 2010.

PASQUALI, L.; AZEVEDO, Maria. M.; GHESTI, Ivânia. Inventário fatorial de personalidade: manual técnico e de avaliação. São Paulo: Casa do Psicólogo, 1997.

PINHEIRO, C. A. R., GOMES, C. M. A.; BRAGA, A. G. Construção e Validação do Inventário soa Adjetivos de Personalidade. In Anais do IV Congresso Brasileiro de Avaliação Psicológica e XIV Conferência Internacional de Avaliação Psicológica: Formas e Contextos. Instituto Brasileiro de Avaliação Psicológica, Campinas, SP, 2009.

PIRES, J. G.; NUNES, C. H. S. S; NUNES, M. F. O. IN: BAPTISTA, Makilim Nunes et al. (org). Compêndio de Avaliação Psicológica. 1 ed. Petrópolis: Editora Vozes, pp. 494-506, 2019.

PRIMI, R. Avaliação psicológica no Brasil: fundamentos, situação atual e futuro para o futuro. Psicologia: teoria e pesquisa, v. 26, n. especial, pp. 25-35, 2010.

REPPOLDI, C. T; ZANINI, D. S.;NORONHA, A. P. P. O que é Avaliação Psicológica?. In: BAPTISTA, Makilin Nunes et al. Compêndio de Avaliação Psicológica. Petrópolis: Editora Vozes, 2019, p. 15-28.

ROTHER, E. T. Revisão sistemática X revisão narrativa. Acta Paulista de Enfermagem, v. 20, n. 2, pp. 5-6, 2007.

SILVEIRA, L. B., OLIVEIRA, S. E. S.; BANDEIRA, D. R. Evidências de validade do inventário de organização da personalidade - Brasil (IPO-Br): relação com o modelo dos cinco grandes fatores da personalidade. Temas psicol., v. 26, n. 4, p. 1875-1889, 2018.

SISTO, F. F et al. Escala de traços de personalidade para crianças e aceitação social pares. Interação em Psicologia, v. 8, n. 1, 2004.

SOTO, C. J.; JOHN, O. P. Short and extra-short forms of the Big Five Inventory-2: the bfi-2-s and bfi-2-xs. Journal Of Research In Personality, [S.L.], v. 68, pp. 69-81, 2017.

SOARES, L., MACHADO, P. P., DIAS, P. PINHO, A.; KLEIN, J. M. Inventário de Personalidade de Freiburg-Revisto (FPI-R): Estudo de validação junto de amostra de estudantes universitários. International Journal of Clinical and Health Psychology, v. 5, n. 2, pp. 319-333, 2005.

TRENTINI, C.. Correlações entre a EFN-escala fatorial de neuroticismo e o IFPinventário fatorial de personalidade. Avaliação Psicológica: Interamerican Journal of Psychological Assessment, v. 8, n. 2, pp. 209-217, 2009. Inovações 
TRÓCCOLI, B. T.; VASCONCELOS, T. S.; PASQUALI, L. ICFP-R-Inventário Reduzido dos Cinco Grandes Fatores de Personalidade: Manual Técnico e de Aplicação. LabPAM, Universidade de Brasília, Brasília, 2004.

VASCONCELlOS, S. J. L.; HUTZ, C. S. Construção e validação de uma escala de abertura à experiência. Avaliaçao Psicologica: Interamerican Journal of Psychological Assessment, v. 7, n. 2, pp. 135-141, 2008.

WECHSLER, S. M; HUTZ, C. S; PRIMI, R. O desenvolvimento da avaliação psicológica no Brasil: Avanços históricos e desafios. Avaliação psicológica. v. 18, n. 2, pp. 121-128, 2019.

ZIEGLER, M. et al. Openness as a buffer against cognitive decline: the openness-fluidcrystallized-intelligence (ofci) model applied to late adulthood. Psychology And Aging, [S.L.], v. 30, n. 3, pp. 573-588, 2015. 